\title{
Clinical Evaluation of Non-functional Invasive Hypophysis Adenomas
}

\author{
Feyzi Gokosmanoglu1, Ceyhun Varim², and Kenan Evren Oztop ${ }^{3}$ \\ ${ }^{1}$ Department of Endocrinology, Medical Park Hospital, Ordu, Turkey \\ ${ }^{2}$ Department of Internal Medicine, Sakarya University Medicine Faculty, Sakarya, Turkey \\ ${ }^{3}$ Department of Internal Medicine, Konak Hospital, Sakarya, Turkey
}

Corresponding Author:

Ceyhun Varim, MD;

Adnan Menderes caddesi,

Sağlık Sokak, No: 195-54000,

Adapazarı/Sakarya.

Tel: +90 2642552106

Fax number: +90 26425521

05.

email:

ceyhunvarim@sakarya.edu.tr

Received 15 April 2020

Accepted 12 June 2020

Published 30 June 2020

Production and Hosting by

Knowledge E

(c) Feyzi Gokosmanoglu et al. This article is distributed under the terms of the

Creative Commons

Attribution License, which permits unrestricted use and redistribution provided that the original author and source are credited.

Editor-in-Chief:

Prof. Mohammad A. M. Ibnouf

\section{Abstract}

Background: There are ongoing studies to predetermine non-functional invasive pituitary adenomas that may show aggressive behavior. Our aim is to discuss whether there is a relationship between the immunohistochemical presence of $\mathrm{GH}, \mathrm{FSH}$, $\mathrm{LH}, \mathrm{PRL}, \mathrm{ACTH}, \mathrm{TSH}$ and their aggressive clinical course in non-functional pituitary adenomas.

Materials and Methods: In this study, we evaluated retrospectively the files of the patients who were diagnosed with the sellar or parasellar tumor in our endocrinology clinic between the years of 2004 and 2014. The patients were divided into two groups as non-invasive pituitary adenomas and non-functional invasive pituitary adenomas. The immunohistochemical staining characteristics were compared between the two groups.

Results: In this study, we scanned the data of 70 patients who were followed for non-functional sellar or parasellar mass; $47.1 \%$ of the patients were female and $52.9 \%$ were male. Of them, 39 patients had a non-functional pituitary adenoma whereas $20.5 \%$ of them had non-functional invasive adenoma. While there was a significant relationship between the immunohistochemical positivity of $\mathrm{GH}, \mathrm{FSH}, \mathrm{LH}$ and the aggressive behavior of non-functional invasive adenomas. no significant relationship was found between the immunohistochemical positivity of PRL, ACTH, TSH and the aggressive behavior of non-functional invasive adenomas.

Conclusion: We found silent $\mathrm{GH}$ and gonadotropin adenomas as non-functional aggressive pituitary adenoma. More aggressive treatment and close clinical monitoring should be performed because atypical pituitary adenomas are characterized by invasive growth and aggressive clinical course.

Keywords: Pituitary adenoma, non-functional, invasive

\section{Introduction}

Hypophysis adenoma, contrary to common belief, is quite widespread. It is found in the normal population at a rate of $20 \%$. Shedding light on the embryological development of adenohypophysis and transcription factors related to molecular cell differentiation 
pathways has led to innovations in the diagnostic approach for adenomas. To that end, a number of different classification models have been developed, which may be classified as functional, anatomic, radiological, histological, immunohistochemical, ultrastructural, or clinicopathological [1].

Invasive hypophysis adenomas are very large, treatment-resistant adenomas that exhibit common and extensive invasion of peripheral anatomic structures while growing rapidly and recurring quickly in the wake of treatment. Their biological behavior falls between hypophysis adenomas and hypophysis cancers. About $2.7 \%$ of hypophysis adenomas are invasive tumors [2]. In 2004, the World Health Organization classified atypical hypophysis adenomas as tumors showing invasive growth and increased mitotic activity, with those having an MIB.1 proliferative index > 3\% and p53 immunoreactivity being atypical [3].

In this study, we aimed to retrospectively evaluate the clinical results of cases of non-functional invasive hypophysis adenoma in patients followed-up within our clinic.

\section{Materials and Methods}

In this study, the medical files of the patients who were followed-up with the diagnosis of sellar and parasellar tumors in the Samsun Ondokuz Mayıs University Medicine Faculty Endocrinology Clinic between the years 2004 and 2014 were retrospectively examined by us. Hypophysis adenomas were separated from the tumoral lesions, which are seen rarely in the other sellar and parasellar regions. Detailed anamnesis and demographic characteristics of the patients, as well as their reasons for presenting at the hospital and the frequency of applications, were recorded from their medical files (Table 1). The clinical and radiological images of the cases and dynamic test data regarding hormones were obtained from the patient files. Adenomas were divided into two groups, namely functional and non-functional. As for hypophysis adenomas, the functional ones were determined according to the hormone content in the foreground and excluded from the study.

TABLE 1: The distribution of the symptoms of the cases at the time of diagnosis.

\section{Symptoms and Findings}

Headache

Visual impairment

Sexual anorexia, infertility

Non-specific symptoms

Asymptomatic, incidental detections
The Number of Patients and Percentages

$34.2 \%(n=34)$

$21,4 \%(n=15)$

$5,7 \%(n=5)$

$12,8 \%(n=9)$

$10 \%(n=7)$ 
TABLE 2: Clinicopathological and immunohistochemical staining results of the patients.

Type of adenoma, immunohistochemical
classification
Hypophysis adenoma, plurihormonal
Cystic lesions
Hypophysis adenoma, staining-negative
Hypophysis adenoma, blood and blood clot
GH (+)
LH (+)
ACTH (+)
Craniopharyngioma
Meningioma
Neurofibroma
Registries missing
Total

\begin{tabular}{|c|c|}
\hline Number of cases \\
\hline 20 \\
12 \\
\hline 8 \\
\hline 7 \\
2 \\
1 \\
1 \\
5 \\
3 \\
1 \\
10 \\
70
\end{tabular}

TABLE 3: Immunohistochemical staining characteristics of non-functional invasive hypophysis adenomas.

Type of adenoma, immunohistochemical
classification
Hypophysis Adenoma, plurihormonal
FSH, LH
PRL
GH
ACTH
TSH
Hypophysis adenoma, staining-negative
Hypophysis adenoma, blood and blood
clot
Total

\begin{tabular}{|c|}
\hline Group 1 \\
\hline 20 \\
\hline 11 \\
\hline 8 \\
\hline 7 \\
\hline 12 \\
\hline 5 \\
\hline 8 \\
\hline 7 \\
\hline 39
\end{tabular}

\begin{tabular}{c} 
Group 2 \\
5 \\
6 \\
3 \\
5 \\
1 \\
- \\
1 \\
- \\
\hline
\end{tabular}

\begin{tabular}{|l|}
\hline P-value \\
\hline 0.013 \\
\hline 0.306 \\
\hline 0.009 \\
\hline 0.298 \\
\hline
\end{tabular}

Group 1: non-invasive adenomas, Group 2: invasive adenomas

TABLE 4: Immunohistochemical classification and distribution of invasive adenomas

\begin{tabular}{|c|}
\hline Type of adenoma \\
\hline $\begin{array}{l}\text { Hypophysis adenoma, } \\
\text { plurihormonal }\end{array}$ \\
\hline \\
\hline \\
\hline $\begin{array}{l}\text { Hypophysis adenoma, } \\
\text { staining-negative }\end{array}$ \\
\hline $\mathrm{GH}(+)$ \\
\hline Total \\
\hline
\end{tabular}

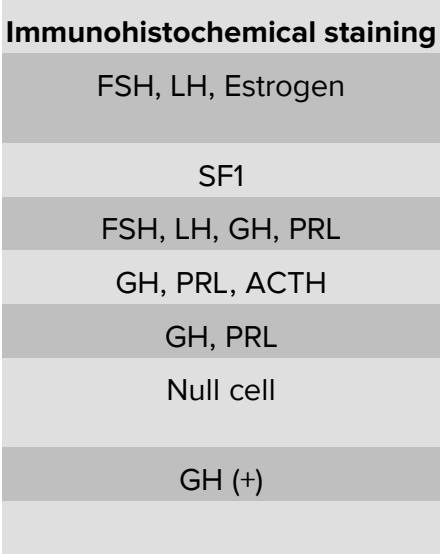

\begin{tabular}{|c|}
\hline Number of cases \\
\hline 2 \\
\hline 1 \\
1 \\
1 \\
\hline 1 \\
\hline 2 \\
\hline 8
\end{tabular}


In our research, sellar and parasellar regional lesions were classified as either nonfunctional hypophysis adenomas or other lesions (cyst, teratoma, metastasis, infectious and inflammatory lesions, glioma, germinoma, neurofibroma, meningioma and craniopharyngioma) using immunohistochemical staining data. Non-functional non-invasive hypophysis adenomas and non-functional invasive hypophysis adenomas were separated into two groups: Group 1 and Group 2. The immunohistochemical staining characteristicsof both groups were compared as well (Table 2).

Hypophysis adenomas were specified as microadenoma $(<10 \mathrm{~mm})$ and macroadenoma ( $\geq 10 \mathrm{~mm}$ ) according to their hypophysis/pituitary sizes on MRI.

All the data were determined as mean $( \pm)$ standard deviation (SD). The statistical analyses were performed through SPSS 16.0 (SPSS Inc., Chicago, IL) programme. The statistical analyses of their mean comparisons were performed via Independent Two Sample $T$-Test and Chi-square Test. Numeric values and percentages of all the determinants were evaluated via frequency analyses. As the $p$-value was $<0.05$ during the comparison process, it was accepted as statistically significant.

\section{Results}

Research was carried out in the Endocrinology Clinic of our hospital between 2004 and 2014 by retrospectively examining the data of 70 patients who were followed-up due to non-functional sellar and parasellar lesions following hypophysis; $47 \%$ of the patients ( $n$ = 33) were female while $53 \%$ of them $(n=37)$ were male. The mean age was 48 years and $90 \%$ of the patients $(n=63)$ were asymptomatic at the time of diagnosis.

In our study, $18.5 \%$ of the patients $(n=13)$ had microadenoma, while $81.4 \%$ of them ( $n=$ 57) had macroadenoma. At the first admission to the hospital, 57 patients were followedup through the treatment process while 13 of them through an unmedicated method. All patients who went through an unmedicated follow-up process had microadenomas. Fifty surgical patients underwent transsphenoidal surgery and seven patients underwent transcranial surgery. A remission rate of $80.7 \%(n=46)$ was detected along with surgical treatment; however, a 19.2\% $(n=11)$ residual tumor rate was detected despite surgical treatment.

During clinicopathological and immunohistochemical examinations of the cases, no record could be found for 55.8\% ( $n=39$ ) of hypophysis adenoma, 30\% $(n=21)$ of cystic lesions, craniopharyngioma, meningioma, neurofibroma, or $14.2 \%(n=10)$ of immunohistochemical staining (Table 3). The clinicopathological and immunohistochemical staining results of 39 cases that were followed-up with a diagnosis of non-functional hypophysis 
adenoma are given in Table 3. A treatment-resistant invasive growth that progressed aggressively was found in eight cases. No remission was achieved, despite the fact that five of these cases had been operated on twice and three of them had been operated on thrice, and the gamma knife treatment was provided during the post-operative period. The immunohistochemical staining results of eight adenomas showing a treatmentresistant invasive growth with an aggressive course are shown in Table 4.

Statistically, we ascertained a significant relationship between $\mathrm{GH}$ presence in the immunohistochemical staining process and invasive and aggressive behavioral patterns of non-functional adenomas $(p=0.009)$. The $\mathrm{FSH}$, LH positivity in adenomas caused adenomas to demonstrate invasive and aggressive behavior $(p=0.013)$. During the immunohistochemical staining process of non-functional hypophysis adenomas, no significant relationship could be found between the presence of PRL, ACTH, TSH and the invasive, aggressive behavioral patterns of adenomas.

\section{Discussion}

The prevalence rate of hypophysis adenomas is approximately $20 \%$ [4]. However, $>90 \%$ of the patients operated on with the diagnosis of hypophysis tumor were diagnosed with hypophysis adenoma [4].

Other kinds of hypophysis tumors are Rathke's cleft cyst (28\%), craniopharyngioma (14\%), metastatic carcinoma (12\%), chordoma (11\%), and meningioma (10\%) [4].

In our research, 55.8\% of the non-functional sellar and parasellar regional tumoral lesions were regarded as hypophysis adenoma in the wake of the operation, whereas $30 \%$ of them were considered as cystic lesions, craniopharyngioma, meningioma, and neurofibroma. The reason for the low percentage in the diagnosis of post-operative hypophysis adenoma in our study may be due to the exclusion of functional adenomas from the study and the missing immunohistochemical staining records in $14.2 \%$ of cases.

Apart from being slowly growing benign tumors, hypophysis adenomas tend to lead to an invasion of the cavernous sinus and even the sphenoid sinus by causing pressure against the optic chiasm after surpassing the sella. It is also possible that invasive adenomas infiltrate bone, and more rarely the brain (5). In our study, all invasive adenomas were macroadenomas, and radiological reports showed that they had invaded the sellar and suprasellar region.

In the literature, the incidence of atypical hypophysis adenoma was reported as ranging from 2.7 to 15\%. [6-8]. In half of these cases, a non-functional hypophysis adenoma was detected [6]. In our research, we detected a treatment-resistant invasive 
adenoma exhibiting invasive growth with an aggressive course in $20.5 \%$ of 39 cases ( $n$ = 8) with non-functional hypophysis adenoma. The fact that we detected a high level of invasive adenoma in non-functional hypophysis adenomas may be explained by the fact that the cases incorporated into the study were complicated and the challenging ones were followed-up through a multidisciplinary approach.

In 2004, the World Health Organization classified hypophysis adenomas as atypical adenoma and carcinoma in accordance with the classification of endocrine tumors. Cases demonstrating an apparent mitotic activity, a Ki-67 proliferative index over $3 \%$, and a common nuclear p53 protein positivity are defined as atypical hypophysis adenoma (3).

Differing from the criteria of the World Health Organization as to atypical adenomas, we determined in our research that positive adenomas had shown an atypical and aggressive course through $\mathrm{GH}$ positivity and $\mathrm{FSH}, \mathrm{LH}$, and immunohistochemical staining. Recurrences and aggressiveness are very rare in cases of Ki-67 proliferative index and p53 protein positivity after surgery [9].

Separately, aggressive behavior can also be seen in the absence of these immunohistochemical findings [9]. It was shown that silent $\mathrm{GH}$ and gonadotroph adenoma tended to be invasive macroadenomas or giant adenomas [10]. Also, in our research we ascertained that silent $\mathrm{GH}$ and gonadotroph adenomas had progressed aggressively.

We detected an estrogen receptor positivity in $25 \%$ of cases with non-functional invasive hypophysis macroadenoma during the immunohistochemical staining process. During the research, estrogen receptor alpha positivity was determined to be prominently higher (80\%) in invasive macroadenomas than in microadenomas (3.33\%) [11]. The estrogen receptor alpha in non-functional invasive adenomas was reported to have been stained more intensely than in the non-invasive ones [12]. The estrogen receptor alpha was shown to have been expressed more in FSH-LH, GH, and null cell adenomas than ACTH adenomas (13). In our study, we demonstrated an estrogen receptor in invasive $\mathrm{FSH}-\mathrm{LH}$ adenomas.

In the literature, $<70 \%$ of atypical adenomas were reported to have formed $\mathrm{GH}$ secreting, null cell, and silent ACTH adenomas. Recently, it has been reported that nonfunctional gonadotropin adenomas exhibit invasive behavioral patterns [6]. However, in our research, silent GH adenoma and non-functional gonadotropin adenomas were seen to have progressed in an aggressive manner. We determined that $\mathrm{ACTH}, \mathrm{TSH}$, and PRL positivity, which are among the histopathological sub-types, and also null cell adenomas act as typical adenomas. 
We ascertained that $20.5 \%$ of non-functional hypophysis adenomas were atypical, invasive macroadenomas that acted aggressively. Silent GH adenomas and gonadotropin adenomas comprised a majority of these tumors. Since atypical hypophysis adenomas are characterized by invasive development and an aggressive clinical course, close clinical follow-up of these cases must be performed in terms of their recurrence or distant metastasis. Contributing to the development of target-specific treatments, we are of the opinion that the sub-types of hypophysis adenomas should be specified by taking current approaches into consideration, and that the treatment and follow-up of particular sub-types should follow aggressive courses that must be performed more carefully.

\section{Conflict of Interest}

The authors declared that there is no conflict of interest

\section{Author Contribution}

FG found the subject and collected data, CV wrote the article and searched the references, and KEO translated the article to English and created the tables.

\section{References}

[1] Asa, S. L. (2008). Practical pituitary pathology. What does the pathologist need to know? Archives of Pathology \& Laboratory Medicine, vol. 132, pp. 1231-1240.

[2] Buchfelder, M. (2009). Management of aggressive pituitary adenomas: current treatment strategies. Pituitary, vol. 12, pp. 256-260.

[3] DeLellis, R., Lloyd, R. V., Heitz, P., et al. (eds). (2004). World Health Organization Classification of Tumours: Tumours of Endocrine Organs, pp. 10-35. Lyon: IARC Press.

[4] Davis, J. R., Farrell, W. E., and Clayton, R. N. (2001). Pituitary tumours. Reproduction, vol. 121, no. 3, pp. 363-371.

[5] Botelho, C. H., Magalhaes, A. V., Mello, P. A., et al. (2006). Expression of p53, Ki67 and c-erb B2 in growth hormoneand/or prolactin-secreting pituitary adenomas. Arquivos de Neuro-Psiquiatria, vol. 64, no. 1, pp. 60-66.

[6] Zada, G., Woodmansee, W. W., Ramkissoon, S., et al. (2011). Atypical pituitary adenomas: incidence, clinical characteristics and implications. Journal of Neurosurgery, 
vol. 114, pp. $336-344$.

[7] Tortosa, F. and Webb, S. M. (2015). Atypical pituitary adenomas: 10 years of experience in a reference centre in Portugal. Neurología, vol. 31, no. 2, pp. 97-105.

[8] Saeger, W., Lüdecke, D. K., Buchfelder, M., et al. (2007). Pathohistological classification of pituitary tumors: 10 years of experience with the German Pituitary Tumor Registry. European Journal of Endocrinology, vol. 156, pp. 203-216.

[9] Gejman, R., Swearingen, B., and Hedley-Whyte, E. T. (2008). Role of Ki-67 proliferation index and p53 expression in predicting progression of pituitary adenomas. Human Pathology, vol. 39, pp. 758-766.

[10] Gomez-Hernandez, K., Ezzat, S., Asa, S. L., et al. (2015). Clinical implications of accurate subtyping of pituitary adenomas: perspectives from the treating physician. Turkish Journal of Pathology, vol. 31, pp. 4-17.

[11] Pereira-Lima, J. F., Marroni, C. P., Pizarro, C. B., et al. (2004). Immunohistochemical detection of estrogen receptor alpha in pituitary adenomas and its correlation with cellular replication. Neuroendocrinology, vol. 79, no. 3, pp. 119-124.

[12] Zhou, W., Song, Y., Xu, H., et al. (2011). In nonfunctional pituitary adenomas, estrogen receptors and slug contribute to development of invasiveness. The Journal of Clinical Endocrinology and Metabolism, vol. 96, no. 8, pp. E1237-E1245.

[13] Manoranjan, B., Salehi, F., Scheithauer, B. W., et al. (2010). Estrogen receptors alpha and beta immunohistochemical expression: clinicopathological correlations in pituitary adenomas. Anticancer Research, vol. 30, no. 7, pp. 2897-2900. 